Communications in Physics, Vol.26, No. 1 (2016), pp. 33-42

DOI:10.15625/0868-3166/26/1/8213

\title{
CONTROLLING OPTICAL BISTABILITY IN A FIVE-LEVEL CASCADE EIT MEDIUM
}

\author{
PHAN VAN THUAN, TA TRAM ANH, LE CANH TRUNG, NGUYEN TIEN DUNG, \\ LUONG THI YEN NGA, DINH XUAN KHOA, LE VAN DOAI, AND NGUYEN HUY BANG ${ }^{\dagger}$ \\ Vinh University, 182 Le Duan Street, Vinh City, Vietnam \\ NGUYEN VAN AI \\ Ky Lam High School, Ky Anh district, Ha Tinh province, Vietnam \\ ${ }^{\dagger} E$-mail: bangnh@vinhuni.edu.vn \\ Received 19 April 2016 \\ Accepted for publication 01 June 2016
}

\begin{abstract}
Optical bistability (OB) of an electromagnetically induced transparency (EIT) medium placed in a conventional unidirectional ring cavity is investigated numerically. The medium is excited by a coupling and probe laser lights via a five-level cascade scheme. It is shown that optical bistabe states with controllable switching threshold intensities and width are established simultaneously in three spectral regions corresponding to EIT windows. The current numerical result is compared to a previous analytical result showing influence of coherence terms neglected in the analytical model.
\end{abstract}

Keywords: optical bistability, electromagnetically induced transparency, coherent optical effects.

Classification numbers: 42.65.Pc.

\section{INTRODUTION}

In the last three decades, we have witnessed a remarkable progress in OB research due to its potential applications in both optical sciences and photonic technology such as all-optical switches, all-optical memories, optical transistors, and all-optical logic gates and processors. In the early years of the OB research using atomic media, a great interest was focused on twolevel atomic media [1-3]. It had been shown that bistable states can be established for certain values of input intensity [2,3]. Although the usual OB behaviors of the two-level atomic system were observed experimentally, relevant applications are still limited because only one optical field was employed for both applying and switching, thereby lack of control for switching intensity thresholds.

(C)2016 Vietnam Academy of Science and Technology 
PHAN VAN THUAN et al.

The advent of EIT [4] provides promising media that make an impetus progress in OB research because of their controllable optical properties such as enhanced Kerr nonlinearity [5], reduced absorption and steeper dispersion for slowing light [6], nonlinear optics at low light levels [7]. Thanks to such controllable optical properties, both switching intensity thresholds and width of OB can be reduced significantly as well as easily controlled.

In the beginning of the OB research concerning EIT media, the basic excitation configurations were the three-level systems [8] in which the OB behaviors can be controlled by either intensity or relative phase of controlling field. Since then, several aspects of the three-level systems used for OB have been extensively investigated [9-16]. Although the three-level EIT configurations opened up some promising applications, they are, however, somehow limited in practice because optical properties are modified solely in a narrow spectral transparent region. Extension from the single- to multi-window EIT medium for OB research is therefore of intensive interest. Particularly, four-level EIT configurations with two controlling fields [17-21] and a Kobrak-Rice five-level system [22] with four controlling fields are introduced. These enable to establish multiwindow $\mathrm{OB}$, but it is difficult to control all coupling fields at the same time.

In order to get multi-window EIT with a simple control, we have recently developed a five-level cascade scheme having closely spaced-hyperfine levels [23] which enhances self-Kerr nonlinearity in the present of EIT [24]. Using this excitation scheme, we have also proposed a multi-channel OB system based on a conventional unidirectional ring cavity [25] by an analytical approach. An input-output relationship for a weak field limit of probe light was derived in an analytical form which is convenient and sufficient for optical bistable devices working at low light intensity. For the case of strong probe light intensity or weak coupling field, however, the analytical result needs further refinement by including the neglected coherent terms among the states $|3\rangle$, $|4\rangle$ and $|5\rangle$. This gap is of particular interest for the current work. We take into account the coherence terms and use numerical method to determine the density matrix element corresponding to the probe transition. The switching threshold intensities and widths are investigated with respect to the coupling light and cooperation parameter of the five-level cascade medium.

\section{THEORETICAL MODEL}

We consider a five-level cascade system as shown in Fig. 1. A probe laser beam, with frequency $\omega_{p}$, drives the transition $|1\rangle \leftrightarrow|2\rangle$ with Rabi frequency $\Omega_{p}$, whereas a coupling laser beam (with frequency $\omega_{c}$ and Rabi frequency $\Omega_{c}$ ) couples simultaneously transitions between the state $|2\rangle$ and three closely-spaced states $|3\rangle,|4\rangle$ and $|5\rangle$.

The frequency detuning of the coupling and probe laser is respectively defined as:

$$
\Delta_{p}=\omega_{p}-\omega_{21}, \Delta_{c}=\omega_{c}-\omega_{32} .
$$

In the framework of the semiclassical theory, using the dipole and rotating wave approximations, density matrix elements $\rho_{i k}$ of the five-level system are governed by the following equations [25]:

$$
\begin{aligned}
& \dot{\rho}_{55}=-\Gamma_{52} \rho_{55}-\frac{i}{2} \Omega_{c} a_{52}\left(\rho_{25}-\rho_{52}\right), \\
& \dot{\rho}_{44}=-\Gamma_{42} \rho_{44}-\frac{i}{2} \Omega_{c} a_{42}\left(\rho_{24}-\rho_{42}\right),
\end{aligned}
$$




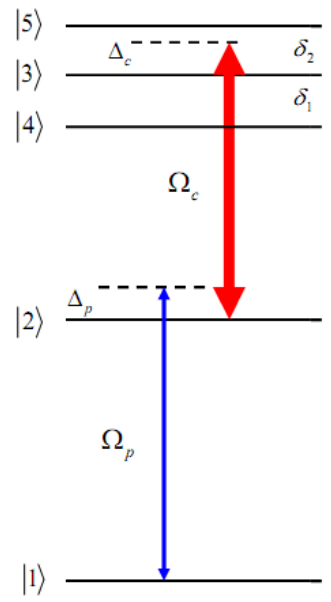

Fig. 1. The five-level cascade system.

$$
\begin{aligned}
\dot{\rho}_{33}= & -\Gamma_{32} \rho_{33}-\frac{i}{2} \Omega_{c} a_{32}\left(\rho_{23}-\rho_{32}\right), \\
\dot{\rho}_{22}= & -\Gamma_{21} \rho_{22}+\Gamma_{32} \rho_{33}+\Gamma_{42} \rho_{44}+\Gamma_{52} \rho_{55}-\frac{i}{2} \Omega_{p}\left(\rho_{12}-\rho_{21}\right)-\frac{i}{2} \Omega_{c} a_{32}\left(\rho_{32}-\rho_{23}\right) \\
& -\frac{i}{2} \Omega_{c} a_{42}\left(\rho_{42}-\rho_{24}\right)-\frac{i}{2} \Omega_{c} a_{52}\left(\rho_{52}-\rho_{25}\right), \\
\dot{\rho}_{11}= & \Gamma_{21} \rho_{22}-\frac{i}{2} \Omega_{p}\left(\rho_{21}-\rho_{12}\right), \\
\dot{\rho}_{54}= & {\left[-i\left(\delta_{1}+\delta_{2}\right)-\gamma_{54}\right] \rho_{54}+\frac{i}{2} \Omega_{c} a_{42} \rho_{52}-\frac{i}{2} \Omega_{c} a_{52} \rho_{24}, } \\
\dot{\rho}_{53}= & {\left[-i \delta_{2}-\gamma_{53}\right] \rho_{53}+\frac{i}{2} \Omega_{c} a_{32} \rho_{52}-\frac{i}{2} \Omega_{c} a_{52} \rho_{23}, } \\
\dot{\rho}_{52}= & {\left[i\left(\Delta_{c}-\delta_{2}\right)-\gamma_{52}\right] \rho_{52}+\frac{i}{2} \Omega_{p} \rho_{51}+\frac{i}{2} \Omega_{c} a_{32} \rho_{53}+\frac{i}{2} \Omega_{c} a_{42} \rho_{54}+\frac{i}{2} \Omega_{c} a_{52}\left(\rho_{55}-\rho_{22}\right), } \\
\dot{\rho}_{51}= & {\left[i\left(\Delta_{c}+\Delta_{p}-\delta_{2}\right)-\gamma_{51}\right] \rho_{51}+\frac{i}{2} \Omega_{p} \rho_{52}-\frac{i}{2} \Omega_{c} a_{52} \rho_{21}, } \\
\dot{\rho}_{43}= & {\left[-i \delta_{1}-\gamma_{43}\right] \rho_{43}-\frac{i}{2} \Omega_{c} a_{42} \rho_{23}+\frac{i}{2} \Omega_{c} a_{32} \rho_{42}, } \\
\dot{\rho}_{42}= & {\left[i\left(\Delta_{c}+\delta_{1}\right)-\gamma_{42}\right] \rho_{42}+\frac{i}{2} \Omega_{p} \rho_{41}+\frac{i}{2} \Omega_{c} a_{32} \rho_{43}+\frac{i}{2} \Omega_{c} a_{52} \rho_{45}+\frac{i}{2} \Omega_{c} a_{42}\left(\rho_{44}-\rho_{22}\right), } \\
\dot{\rho}_{41}= & {\left[i\left(\Delta_{c}+\Delta_{p}+\delta_{1}\right)-\gamma_{41}\right] \rho_{41}+\frac{i}{2} \Omega_{p} \rho_{42}-\frac{i}{2} \Omega_{c} a_{42} \rho_{21}, } \\
\dot{\rho}_{32}= & {\left[i \Delta_{c}-\gamma_{32}\right] \rho_{32}+\frac{i}{2} \Omega_{p} \rho_{31}+\frac{i}{2} \Omega_{c} a_{32}\left(\rho_{33}-\rho_{22}\right)+\frac{i}{2} \Omega_{c} a_{42} \rho_{34}+\frac{i}{2} \Omega_{c} a_{52} \rho_{35}, } \\
\dot{\rho}_{31}= & {\left[i\left(\Delta_{c}+\Delta_{p}\right)-\gamma_{31}\right] \rho_{31}+\frac{i}{2} \Omega_{p} \rho_{32}-\frac{i}{2} \Omega_{c} a_{32} \rho_{21}, }
\end{aligned}
$$




$$
\begin{aligned}
\dot{\rho}_{21} & =\left[i \Delta_{p}-\gamma_{21}\right] \rho_{21}+\frac{i}{2} \Omega_{p}\left(\rho_{22}-\rho_{11}\right)-\frac{i}{2} \Omega_{c} a_{32} \rho_{31}-\frac{i}{2} \Omega_{c} a_{42} \rho_{41}-\frac{i}{2} \Omega_{c} a_{52} \rho_{51}, \\
\rho_{k i} & =\rho_{i k}^{*}, \\
\rho_{11} & +\rho_{22}+\rho_{33}+\rho_{44}+\rho_{55}=1 .
\end{aligned}
$$

where $d_{i k}$ represents the dipole moment of the $|i\rangle-|k\rangle$ transition; $a_{32}=d_{32} / d_{32}, a_{42}=d_{42} / d_{32}$, and $a_{52}=d_{52} / d_{32}$ are the relative transition strengths; $\gamma_{i k}$ represents the decay rate of the density matrix element (coherence) $\rho_{i k}$, given by [24]:

$$
\gamma_{i k}=\frac{1}{2}\left(\sum_{E_{j}<E_{i}} \Gamma_{i j}+\sum_{E_{l}<E_{k}} \Gamma_{k l}\right)
$$

with $\Gamma_{i k}$ being the decay rate of population from level $|i\rangle$ to level $|k\rangle$. We assume a medium of length $L$ composed of $N$ five-level cascade systems placed in a unidirectional ring cavity as shown in Fig. 2, which is the same as introduced in Ref. [25]. For simplicity, we assume both mirrors 3 and 4 are perfectly reflective whereas both mirrors 1 and 2 are the same, each has a reflectivity $R$ and transmitivity $T$, with $R+T=1$.

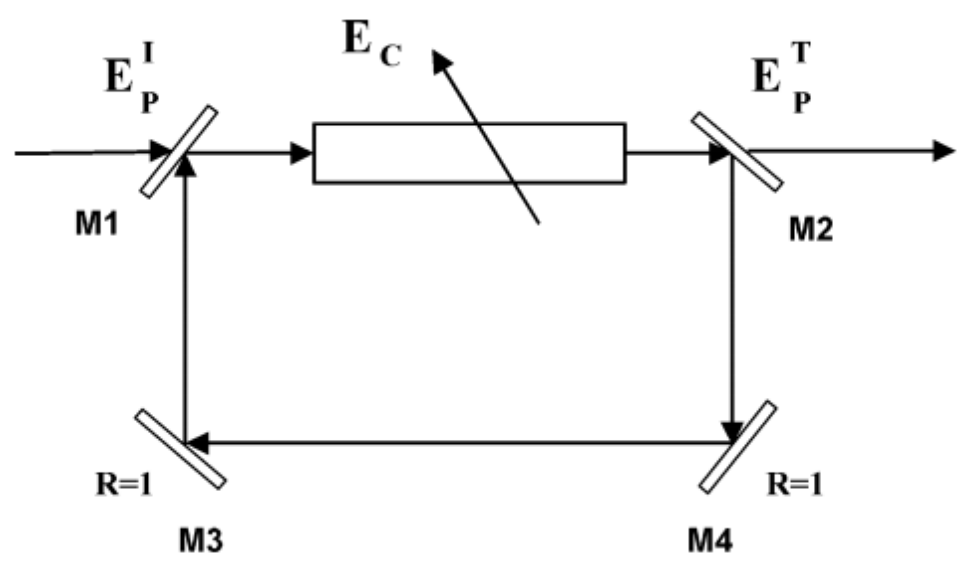

Fig. 2. Schematic setup of a indirectional ring cavity containing a five-level sample; $E_{p}^{I}$ and $E_{p}^{T}$ denote the incident and transmitted probe field, respectively.

The total electromagnetic field can be written as

$$
E=E_{p} e^{-i \omega_{p} t}+E_{c} e^{-i \omega_{c} t}+c . c .,
$$

In the ring cavity, part of the probe field $E_{p}$ is circulated in the ring cavity but the coupling field $E_{c}$ is not. Under the slowly varying envelop approximation, the dynamic response of the probe field governed by Maxwell equations is given by [2]:

$$
\frac{\partial E_{p}}{\partial t}+c \frac{\partial E_{p}}{\partial z}=i \frac{\omega_{p}}{2 \varepsilon_{0}} P\left(\omega_{p}\right),
$$


where $c$ and $\varepsilon$ are the speed of light and permittivity of free space, respectively; $P\left(\omega_{p}\right)$ is the slowly oscillating term of the induced polarization in the transition $|1\rangle \leftrightarrow|2\rangle$ given by:

$$
P\left(\omega_{p}\right)=N d_{21} \rho_{21} \text {. }
$$

Substituting Eq. (22) into Eq. (21), we obtain the following relation:

$$
\frac{\partial E_{p}}{\partial z}=i \frac{N \omega_{p} d_{21}}{2 c \varepsilon_{0}} \rho_{21} \text {. }
$$

For a single circulation of the probe field in the cavity, we denote the probe field at the beginning and the end of the sample by $E_{p}(0)$ and $E_{p}(L)$, respectively (see Fig. 2). For a perfectly tuned cavity, the boundary conditions in the steady state for the incident and transmitted probe fields are given by [2]:

$$
\begin{gathered}
E_{p}(L)=E_{p}^{T} / \sqrt{T}, \\
E_{p}(0)=\sqrt{T} E_{p}^{T}+R E_{p}(L) .
\end{gathered}
$$

By normalizing the incident and transmitted probe field as

$$
Y=\frac{d_{21} E_{p}^{I}}{\hbar \sqrt{T}}, \quad X=\frac{d_{21} E_{p}^{T}}{\hbar \sqrt{T}}
$$

we obtain the following input-output relation for the probe field:

$$
Y=X-i C \rho_{21},
$$

where

$$
C=\frac{N \omega_{p} L d_{21}^{2}}{2 c \varepsilon_{0} \hbar T}
$$

is the cooperation parameter of the five-level medium placed in the unidirectional ring cavity.

\section{NUMERICAL RESULTS AND DISCUSSIONS}

In order to study the behavior of the input-output relation for the probe field via Eq. (26), we determine the atomic coherence $\rho_{21}$ by solving numerically the set of density matrix equations of motion, from Eq. (2) to Eq. (18), in the steady regime. As similar to Ref. [25], we consider the case of ${ }^{85} \mathrm{Rb}$ atomic vapor in which the states $|1\rangle,|2\rangle,|3\rangle,|4\rangle$, and $|5\rangle$ are chosen as $5 \mathrm{~S}_{1 / 2}(F=3), 5 \mathrm{P}_{3 / 2}\left(F^{\prime}=3\right), 5 \mathrm{D}_{5 / 2}\left(F^{\prime \prime}=3\right), 5 \mathrm{D}_{5 / 2}\left(F^{\prime \prime}=4\right)$, and $5 \mathrm{D}_{5 / 2}(F "=2)$, respectively. The atomic parameters for ${ }^{85} \mathrm{Rb}$ are given by (the decay constants and linewidths are given in units of $2 \pi$ ) [26]: $\delta_{1}=9 \mathrm{MHz}, \delta_{2}=7.6 \mathrm{MHz}, \Gamma_{21}=6 \mathrm{MHz} ; \Gamma_{32}=\Gamma_{42}=\Gamma_{52}=\gamma=0.97 \mathrm{MHz}$, $d_{21}=4.5 \times 10^{-18}$ esu.cm, and $a_{31}: a_{42}: a_{52}=1: 1.46: 0.6$.

In the first step, we investigate the variation of output intensity $|X|$ versus input intensity $|Y|$ and frequency detuning $\Delta_{p}$ for the probe field. Here, values of intensity and frequency detuning of coupling field are respectively fixed at $\Omega_{c}=10 \gamma$ and $\Delta_{c}=0$ at which the self-Kerr nonlinearity of the system is enhanced significantly in the three EIT windows (see Ref. [24]). A surface plot of input intensity $|X|$ as a function of variables $|Y|$ and $\Delta_{p}$ for the probe field is shown in Fig. 3. The figure shows OB in three spectral regions that correspond to three EIT windows (see Ref. [23]). Such simultaneous occurrence of $\mathrm{OB}$ at a given value of input intensity provides a possible way to create a multi-channel OB. For a detail, we plot the input-output intensity relation at several values of frequency detuning, as in Fig. 3(d). Here, we introduce the switching threshold intensities $Y_{1}$ 
(for the lower branch) and $Y_{2}$ (for the upper branch) which can be determined by solving the differential equation $d Y / d X=0$.
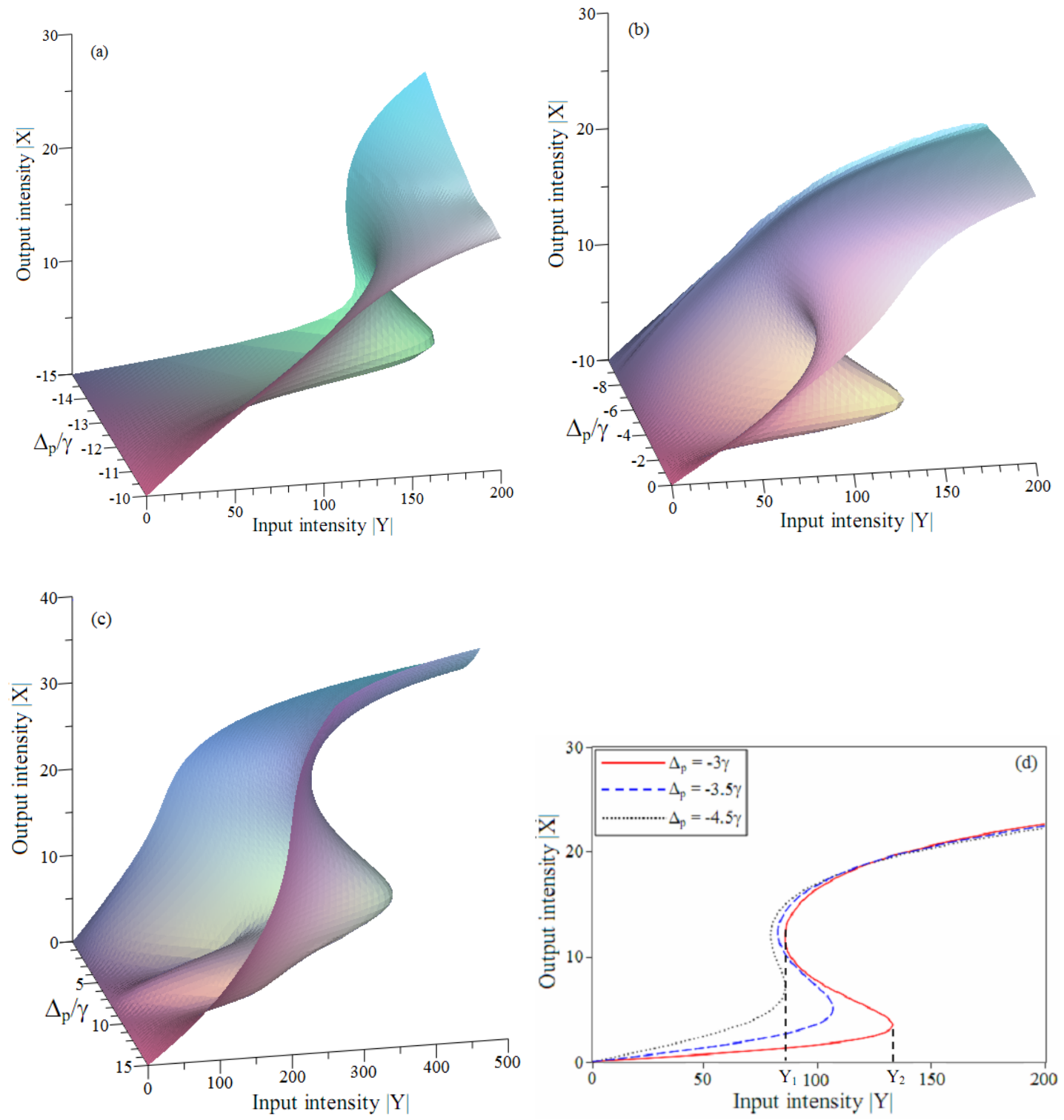

Fig. 3. Surface plots of input-output intensity relation versus frequency detuning $\Delta_{p}$ for the probe field at fixed values: $\Omega_{c}=10 \gamma, \Delta_{c}=0$, and $C=1000 \gamma$. The plots show OB in three spectral regions corresponding to the coupling transitions between states $|2\rangle \leftrightarrow|4\rangle$, $|2\rangle \leftrightarrow|3\rangle$, and $|2\rangle \leftrightarrow|5\rangle$, respectively. Fig. 3(d) represents a particular case of Fig. 3(b) that illustrates dependence of switching thresholds on the probe frequency detuning. 
In order to study the influence of frequency detuning of the coupling light on OB behavior we plot the input-out intensity relationship at $\Omega_{c}=10 \gamma$ and several values of $\Delta_{c}$, as in Fig. 4(a). It is shown that the switching thresholds $\left(Y_{1}\right.$ and $\left.Y_{2}\right)$ and width $\left(Y_{2}-Y_{1}\right)$ of OB can be controlled with $\Delta_{c}$. This is due to sensitive dependence of linear [23] and nonlinear [24] indexes of refraction on the coupling frequency detuning. For further detail, we plot the ratio of switching thresholds $Y_{2} / Y_{1}$ with respect to $\Delta_{c}$ at $\Omega_{c}=10 \gamma$ and $\Omega_{c}=20 \gamma$, as shown in Fig. 4(b). The figure shows that with increasing $\Delta_{c}$ the ratio first grows to a maximum but then drops. An important point here is the possibility to choose frequency detuning $\Delta_{c}$ at a given value of coupling intensity to get "zero width" i.e., $Y_{2} / Y_{1}=1$.
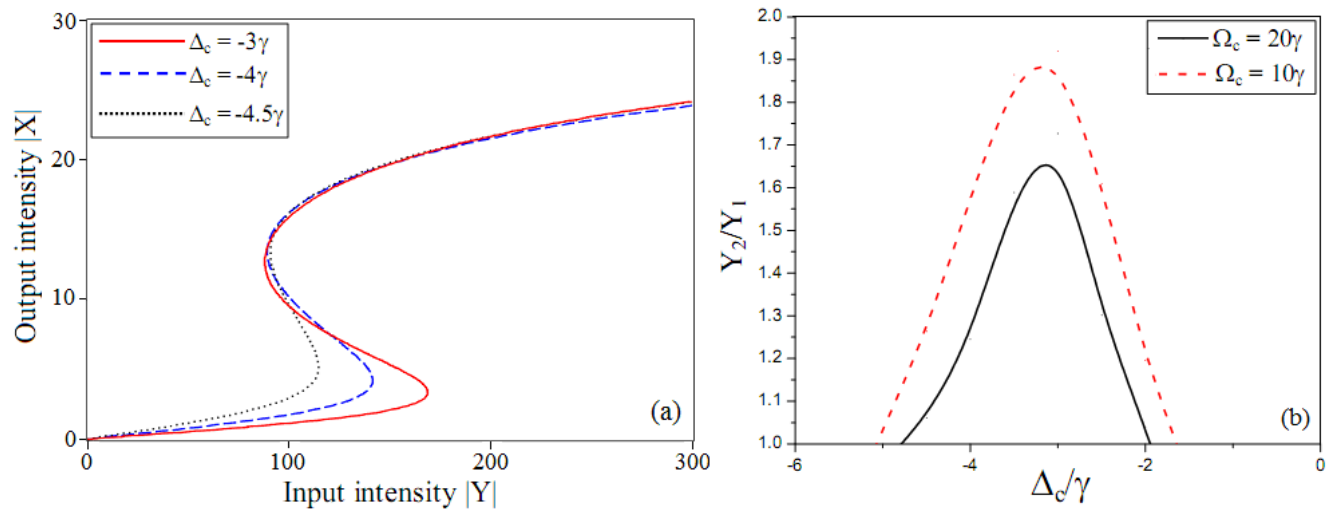

Fig. 4. (a) The input- output relationship at different values of $\Delta_{c}$ when $\Omega_{c}=10 \gamma, \Delta_{p}=0$, and $C=1000 \gamma$; (b) Variation of the ratios of switching intensities $Y_{2} / Y_{1}$ with respect to $\Delta_{c}$ at different values of $\Omega_{c}$ when $\Delta_{p}=0$ and $C=1000 \gamma$.
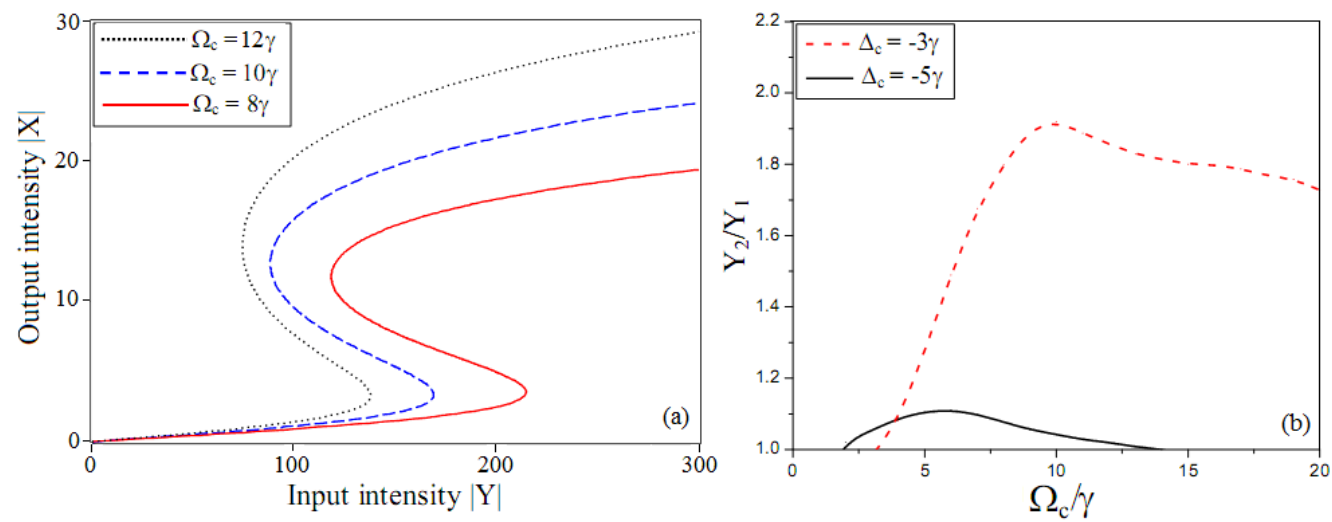

Fig. 5. (a) The input-output relationship at different values of $\Omega_{c}$ when $\Delta_{c}=-3 \gamma, \Delta_{p}=0$ and $\mathrm{C}=1000 \gamma$; (b) Variation of the ratio of switching intensities $Y_{2} / Y_{1}$ with respect to $\Omega_{c}$ when $\Delta_{p}=0$ and $C=1000 \gamma$.

The influence of coupling intensity $\Omega_{c}$ on the OB behavior is also studied by keeping $\Delta_{c}$ $=-3 \gamma$, as shown in Fig. 5(a) and Fig. 5(b). It is shown that one may get "zero width" at various 
values of $\Omega_{c}$ and $\Delta_{c}$. Furthermore, a lower threshold with a "zero width" is found at a higher value of $\Omega_{c}$ (Fig. 5(b)).

In addition to influences of coupling light, influence of the cooperative parameter $C$ on OB is also studied at $\Delta_{p}=0, \Omega_{c}=10 \gamma$, and $\Delta_{c}=-3 \gamma$, as shown in Fig. 6. It is apparent to see that growing of cooperative parameter leads to increasing the width of switching thresholds and lowering output intensity of the upper branch of OB curve. Physically, a growing of $C$ leads to an increase of absorption for the probe light, thus lowering the upper OB branch (output) and requiring higher switching threshold intensity. As a consequence, a "zero width" is only found at a small value of $C$.
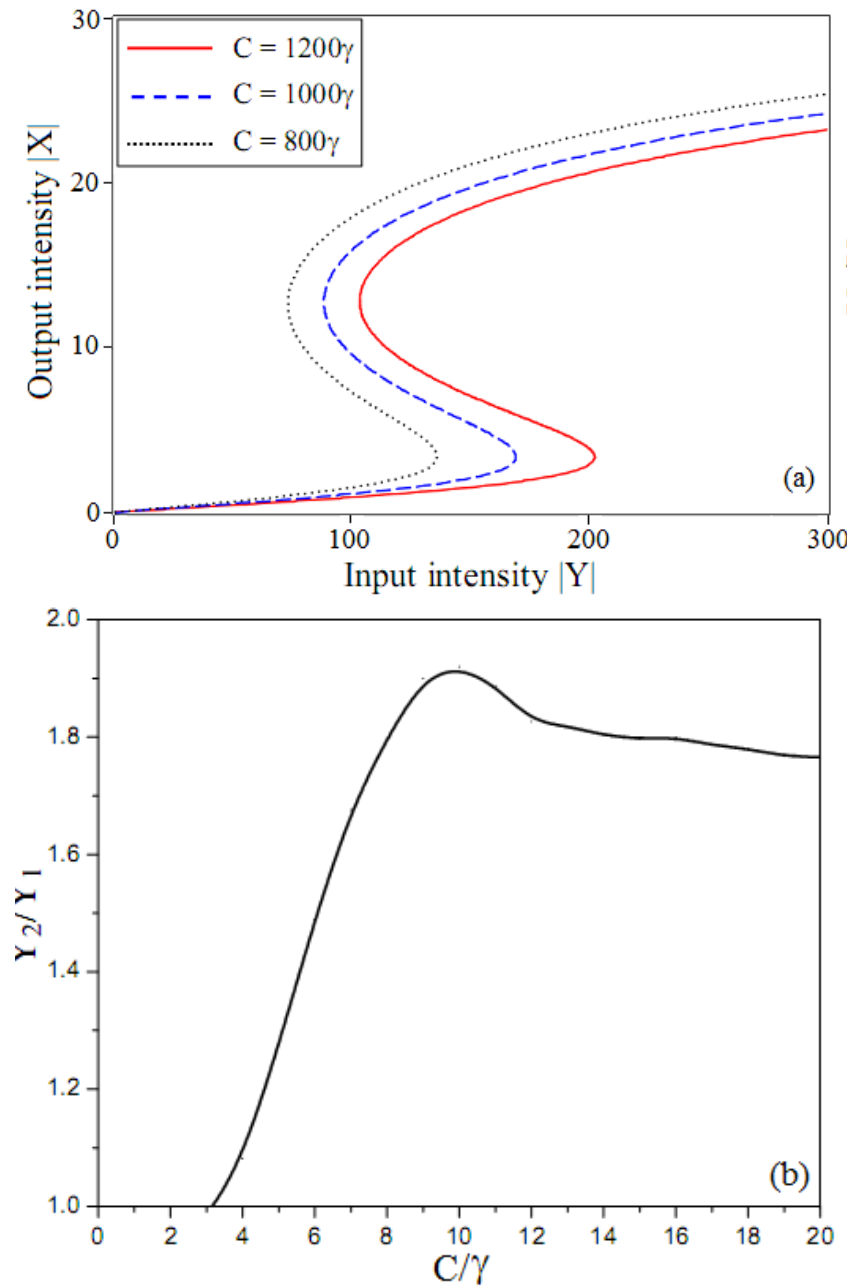

Fig. 6. (a) The input-output relationship at different values of the parameter $C$ when $\Delta_{p}=$ $0, \Omega_{c}=10 \gamma$, and $\Delta_{c}=-3 \gamma$; (b) Variation of the ratios of switching intensities $Y_{2} / Y_{1}$ with respect to $C$ when $\Delta_{p}=0, \Omega_{c}=10 \gamma$, and $\Delta_{c}=-3 \gamma$. 

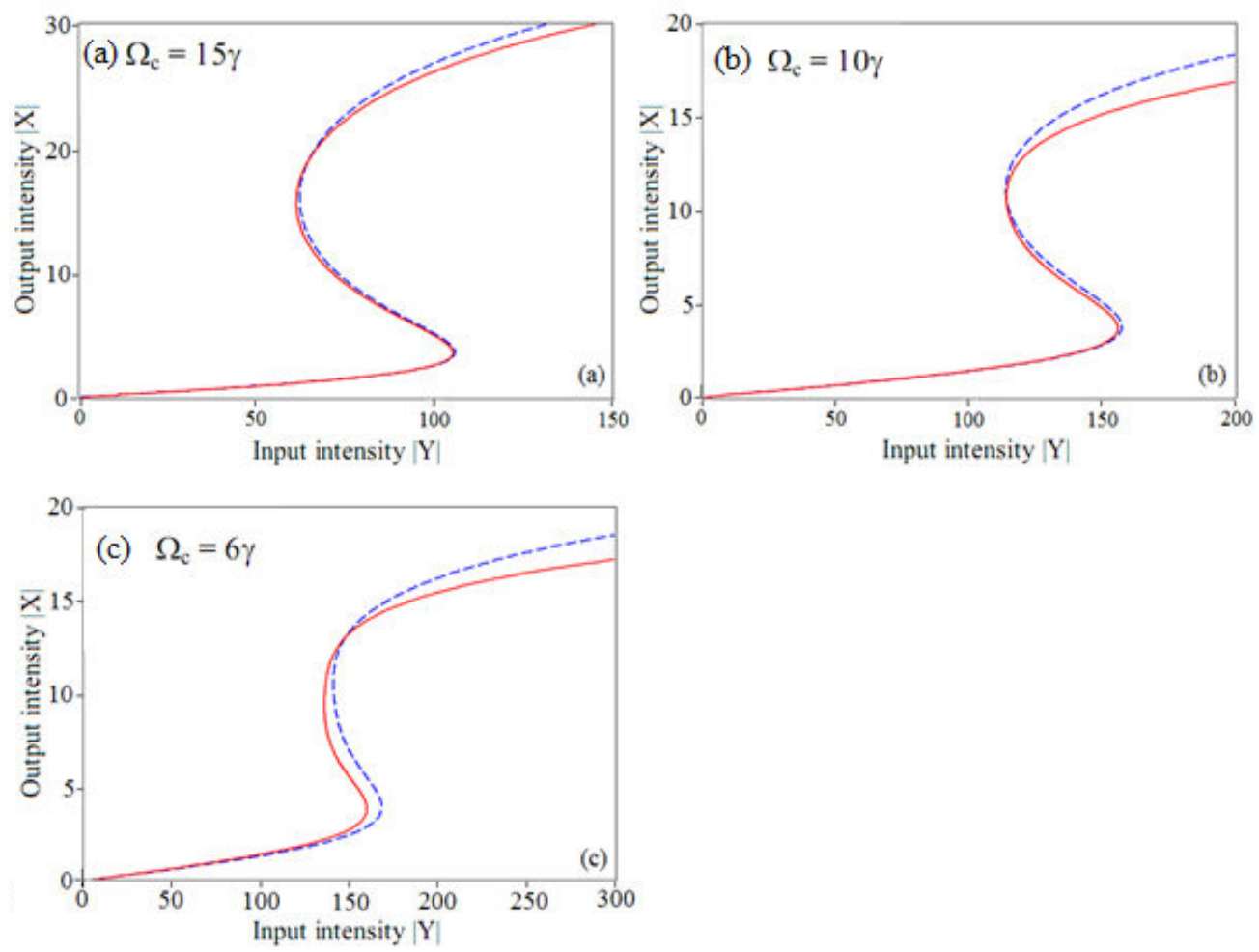

Fig. 7. Comparison between the current numerical OB (dashed curve) with the previous analytical OB obtained in Ref. [25] (solid curve) at different values of the coupling intensity: $(a)-\Omega_{c}=15 \gamma,(\mathrm{b})-\Omega_{c}=10 \gamma$, and (c) $-\Omega_{c}=6 \gamma$. The other papermeters used in the three panels are $\Delta_{p}=-3 \gamma, \Delta_{c}=0$.

Finally, we compare the current numerical results (dashed curve) with the corresponding analytical ones (solid curve) obtained in Ref. [25], as shown in Fig. 7. The comparison shows a minor deviation between the numerical and analytical results for strong coupling, e.g., $\Omega_{c}=15 \gamma$ (Fig. 7(a)). However, the discrepancy increases when lowering the coupling light intensity, see Fig. 7(b) (for $\Omega_{c}=10 \gamma$ ) and Fig. 7(c) (for $\Omega_{c}=6 \gamma$ )". Such deviations show a growing influence of coherent terms which were neglected in the analytical method in Ref. [25], when lowering the coupling light intensity.

\section{CONCLUSIONS}

Using numerical method, we have developed an OB in a five-level cascade EIT medium placed in a conventional unidirectional ring cavity which is controlled solely by a coupling light. The current work takes into account the previously neglected coherent terms to further precise interpretation of $\mathrm{OB}$ in the case of weak intensity of the controlling light. It has been shown that the OB can be established in three spectral regions. Specially, one may choose the frequency detuning and/or intensity of the coupling light and the cooperation parameter $C$ such that the width of the OB switching thresholds shrinks to zero. 
PHAN VAN THUAN et al.

\section{ACKNOWLEDGMENT}

The financial supports from Vietnam's Ministry of Education and Training under the grants coded as 08/2012/H-HTQTSP and B2014-27-14 are acknowledged.

\section{REFERENCES}

[1] E. Abraham and S. Smith, Reports on Progress in Physics 45 (8) (1982) 815.

[2] L. A. Lugiato, Progress in Optics 21 (1984) 69-216.

[3] H. Gibbs, Optical bistability: Controlling light with light, Elsevier, 2012.

[4] K.-J. Boller, A. Imamoğlu, and S. E. Harris, Physical Review Letters 66 (20) (1991) 2593.

[5] H. Schmidt and A. Imamoglu, Optics letters 21 (23) (1996) 1936-1938.

[6] L. V. Hau, S. E. Harris, Z. Dutton, and C. H. Behroozi, Nature 397 (6720) (1999) 594-598.

[7] S. Harris and L. V. Hau, Physical Review Letters 82 (23) (1999) 4611.

[8] S.-q. Gong, Z.-z. Xu, S.-h. Pan, et al., Physics Letters A 222 (4) (1996) 237-240.

[9] H. Wang, D. Goorskey, and M. Xiao, Physical Review A 65 (1) (2001) 011801.

[10] H. Wang, D. Goorskey, and M. Xiao, Optics letters 27 (15) (2002) 1354-1356.

[11] M. Antón and O. G. Calderón, Journal of Optics B: Quantum and Semiclassical Optics 4 (2) (2002) 91.

[12] A. Joshi and M. Xiao, Physical review letters 91 (14) (2003) 143904.

[13] A. Joshi, A. Brown, H. Wang, and M. Xiao, Physical Review A 67 (4) (2003) 041801.

[14] J. Li, Physica D: Nonlinear Phenomena 228 (2) (2007) 148-152.

[15] Z. Wang, A.-X. Chen, Y. Bai, W.-X. Yang, and R.-K. Lee, JOSA B 29 (10) (2012) 2891-2896.

[16] A. Joshi and M. Xiao, Controlling steady-state and dynamical properties of atomic optical bistability, World Scientific, 2012.

[17] J.-H. Li, X.-Y. Lü, J.-M. Luo, and Q.-J. Huang, Physical Review A 74 (3) (2006) 035801.

[18] L. Xin-You, L. Jia-Hua, L. Ji-Bing, and L. Jin-Ming, Journal of Physics B: Atomic, Molecular and Optical Physics 39 (24) (2006) 5161.

[19] M. Sahrai, S. Asadpour, H. Mahrami, and R. Sadighi-Bonabi, Journal of Luminescence 131 (8) (2011) 1682-1686.

[20] M. Sahrai, H. Hamedi, and M. Memarzadeh, Journal of Modern Optics 59 (11) (2012) 980-987.

[21] H. Hamedi, S. Asadpour, M. Sahrai, B. Arzhang, and D. Taherkhani, Optical and Quantum Electronics 45 (3) (2013) 295-306.

[22] L. Ebrahimi Zohravi, R. Doostkam, S. M. Mousavi, and M. Mahmoudi, Progress In Electromagnetics Research M 25 (2012) 1-11.

[23] L. Van Doai, P. Van Trong, D. X. Khoa, and N. H. Bang, Optik-International Journal for Light and Electron Optics 125 (14) (2014) 3666-3669.

[24] D. X. Khoa, L. Van Doai, D. H. Son, and N. H. Bang, JOSA B 31 (6) (2014) 1330-1334.

[25] D. X. Khoa, L. Van Doai, P. Van Thuan, N. T. Dung, N. H. Bang, et al., JOSA B 33 (4) (2016) 735-740.

[26] D. A. Steck, $R b^{85} D$ Line Data, http://steck.us/alkalidata. 